\title{
La segunda pantalla televisiva: la aplicación Ant 3.0 de Antena 3
}

\section{TV Second Screen: Ant 3.0 App by Antena 3}

\author{
Mónica Barrientos-Bueno \\ Profesora Ayudante Doctora (Departamento de Comunicación Audiovisual, \\ Publicidad y Literatura, Universidad de Sevilla)
}

Fecha de recepción: 7 de febrero de 2013

Fecha de revisión: 30 de mayo de 2013

Para citar este artículo: Barrientos-Bueno, M. (2013): La segunda pantalla televisiva: la aplicación Ant 3.0 de Antena 3, Icono 14, volumen 11 (2), pp. 357-383. doi: 10.7195/ri14.v11i2.568 


\section{Resumen}

Dentro del nuevo modelo comunicativo caracterizado por la convergencia multimedia, las aplicaciones de segunda pantalla televisiva para dispositivos móviles están conociendo un lento crecimiento en España. La de mayor implantación y alcance actualmente es Ant 3.0, de ahí su interés como objeto de análisis. Por ello este artículo propone conocer el funcionamiento y principales servicios que ofrece la primera aplicación de contenido sincrónica en España desde la praxis, previo acercamiento al concepto de actividad de segunda pantalla. Por ello el trabajo de campo ha supuesto seguir una sesión de segunda pantalla, para lo cual se ha escogido la emisión de un capítulo de la serie Gran Hotel. Como resultado, Ant 3.0 queda definida como una experiencia de segunda pantalla completa de contenido interactivo que fideliza al espectador y le hace partícipe, en este caso, de la resolución de los misterios de la trama.

\section{Palabras clave}

Televisión - Segunda pantalla - Antena 3 - Aplicación - Ant 3.0 - Dispositivo móvil Interactividad

\section{Abstract}

Inside of the new communication model determined by the multimedia convergence, TV second screen apps for mobile devices are increasing slowly in Spain. The one with higher implantation and range is Ant 3.0, hence its interest to be analyzed. This paper invites to know, from the practice, how it works and the main services provided by the first contents synchronized app in Spain after having an approach to the concept of second screen activity. So the field work has lied in following a second screen session and a broadcast of Gran Hotel series episode has been chosen. As a result, Ant 3.0 app gets defined as a complete second screen experience with interactive contents that develops the spectator's loyalty and makes him being involved, in this case, in solving the mysteries of the plot. 


\section{Key Words}

Television - Second Screen - Antena 3 - App - Ant 3.0 - Mobile Device - Interactivity

\section{Introducción}

A ningún buen observador es ajeno un gran cambio vivido: hoy en día el acto mismo de "ver la televisión" se ha transmutado; no es lo que era hace unos años, los distintos dispositivos electrónicos e informáticos que siembran las actividades diarias de los espectadores participan en ello como producto de la convergencia del medio. Ordenadores, tabletas, smartphones, iPads y iPhones no se emplean únicamente para el desarrollo de la actividad profesional, educativa y lúdica, se han convertido en extensiones de la "primera pantalla", la del televisor. El actual entorno digital, en el que el televidente está inmerso y donde recibe una lluvia de estímulos y datos, hace que demande más de lo que ve con la parrilla de programación en el televisor de su hogar: quiere más capacidad de acción y, además, tiene a su alcance dispositivos tecnológicos que lo hacen posible. De esta manera las aplicaciones de segunda pantalla diseñadas para ellos son una respuesta: ponen a su disposición contenidos exclusivos, ampliación de la información, profundización en el perfil de los personajes de la serie que esté siguiendo en ese momento, datos anecdóticos, curiosidades, breves avances, etc.

Las cadenas de televisión españolas no son indiferentes a ello: Antena 3 y Televisión Española (TVE) están dando los primeros pasos con aplicaciones sincrónicas de segunda pantalla, Ant 3.0 y "Más Isabel", respectivamente. Aunque la primera de ellas es genérica y abarca tanto series como diferentes programas de la cadena, mientras que la segunda es específica para la serie Isabel, ambas tienen en común la ampliación de la experiencia del espectador de series especialmente, género en el que ha arraigado con más fuerza dadas su potencialidad y posibilidades en ese sentido. Por ello cobra gran interés conocer el funcionamiento y contenidos ofrecidos por una aplicación de segunda pantalla implantada en España hace ya un año, como es el caso de Ant 3.0.

Sobre la segunda pantalla televisiva, una serie de recientes informes realizados en el entorno británico por Red Bee Media (2012) ofrecen un acercamiento al concepto y sus implicaciones, acompañado además de un estudio sociodemográfico y

DOI: ri14.v11i2.568 | ISSN: 1697-8293 | Año 2013 Volumen 11 N² 2 | ICONO14 
de hábitos de consumo de la second screen y el potencial comercial que encierra, aún por aprovechar por parte de las cadenas, plataformas y proveedores de contenido. Igualmente otro informe, publicado por JWT Intelligence (Pallete, Jiménez, Paley \& Mack, 2012), aporta datos concretos y estadísticas actualizadas sobre los usos del móvil como vía de consumo de contenidos audiovisuales.

En el área de la investigación científica, la segunda pantalla abre varias perspectivas de estudio, ya se trate del análisis de la atención visual de estas aplicaciones durante el visionado de programas de televisión (Holmes, Josephson \& Carney, 2012), los patrones de comportamiento en el empleo exclusivo de tabletas para el disfrute de estas aplicaciones mientras se ve la televisión (Courtois \& D’Heer, 2012), el diseño de arquitecturas concretas de segunda pantalla que potencien la experiencia interactiva (Cesar, Bulterman \& Jansen, 2009), la segunda pantalla como puerta de la interacción con medios digitales en el entorno doméstico (Tsekleves, Cruickshank, Hill, Kondo \& Whitman, 2007), y los géneros televisivos en relación al diseño de sistemas de televisión social (Geerts, Cesar \& Bulterman, 2008).

El objetivo principal de esta investigación ha sido conocer el funcionamiento de la primera aplicación sincrónica de segunda pantalla en España, puesta en marcha por Antena 3 Televisión: Ant 3.0. De forma particular, se fijaron una serie de objetivos secundarios: definir y acotar lo que es la segunda pantalla televisiva, situar Ant 3.0 en el ámbito de la convergencia multimedia que caracteriza el modelo comunicativo actual, conocer la tecnología específica empleada por la aplicación sincrónica de Antena 3, describir los componentes de la aplicación a través del análisis y descripción de una sesión en particular, la cual sirva como ejemplo representativo del formato y sus contenidos ofertados al espectador.

\section{Material y método}

En la consecución de sus objetivos, la investigación que se presenta planteó dos partes esenciales: teórica y práctica. Para la primera se realizó una síntesis de los principales estudios y análisis sobre la televisión actual y los cambios en los que está inmersa, lo cual ha proporcionado el marco contextual necesario para el adecuado desarrollo de un acercamiento a la creciente implantación de aplicaciones y servicios de segunda pantalla, los cuales se han perfilado a través de literatura específica de reciente publicación dado lo novedoso del auge de la second screen.

ICONO14 | Año 2013 Volumen 11 N² 2 | ISSN: 1697-8293 | DOI: ri14.v1 1i2.568 
El trabajo de campo de la investigación, la de seguimiento de una sesión de segunda pantalla con Ant 3.0, se realizó con varios programas y se ha escogido como ejemplo de caso, por lo completo de la experiencia, contenidos generados y ser uno de los géneros que mejor funcionan con esta tecnología, la emisión del capítulo 18 de la serie Gran Hotel (producida por Bambú Producciones, en antena desde octubre de 2011) con el que se estrenaba la tercera temporada. La experiencia se materializó con un dispositivo Android, un smartphone Samsung Galaxy Ace GT-S5839i, con el cual además se realizaron capturas de pantalla que se incluyen como ilustraciones.

\section{Resultados}

Estamos inmersos en un cambio del patrón comunicativo televisivo, un tercer modelo televisivo de integración entre la informática y las telecomunicaciones, entre televisión e internet (Cebrián Herreros, 2004), impulsado por la evolución tecnológica inherente al medio, el cual ha desembocado en un entorno digital y en alta definición donde la multiplicidad de dispositivos ha conducido, a su vez, a una convergencia cultural multimedia. Es una gran evolución que viene ligada, además de a condiciones tecnológicas, a factores sociales y la intervención de las industrias culturales.

Cuestiones como la interactividad, la hipertextualidad, la digitalización, la virtualidad, la conectividad, la convergencia y portabilidad, entre otras, constituyen algunos de los rasgos técnicos más notables del flamante sistema de medios que los humanos estamos creando para desarrollar nuestras comunicaciones -en el marco de la nueva cultura digital y de nuevas modalidades de sociabilidad e institucionalidad (Aguilera Moyano, 2009).

Entre las distintas pantallas (televisor, ordenador, smartphone, tableta y consola de videojuegos), los teléfonos móviles y tabletas ofrecen, además de movilidad, la posibilidad de conectividad permanente y ubicua gracias a las redes $3 \mathrm{G}$ y WiFi de banda ancha.

Una de las mayores ventajas del móvil es, sin duda, haber conquistado tiempos y espacios hasta el momento no adscritos al consumo mediático tradicional (desplazamientos, momentos de espera, etc.). La ubicuidad, la capacidad de conexión permanente y la posibilidad de llegar al usuario de forma personalizada, señalan

DOI: ri14.v11i2.568 | ISSN: 1697-8293 | Año 2013 Volumen 11 N² 2 | ICONO14 
de forma relevante la especificidad del móvil frente a otros medios y sitúan a la plataforma móvil en un lugar estratégico para dar cabida a un amplio conjunto de servicios y contenidos relacionados con la publicidad, la información y el entretenimiento (Adelantado \& Martí, 2011).

Un reciente informe, 15 ways mobile will change our lives, editado por JWT Intelligence, apunta algunos hábitos de consumo interesantes respecto a los teléfonos móviles que se dan también con otros dispositivos: en España, el $72 \%$ de los usuarios de smartphones desarrollan otras actividades al mismo tiempo que lo usan, las cuales se distribuyen como muestra el gráfico siguiente:

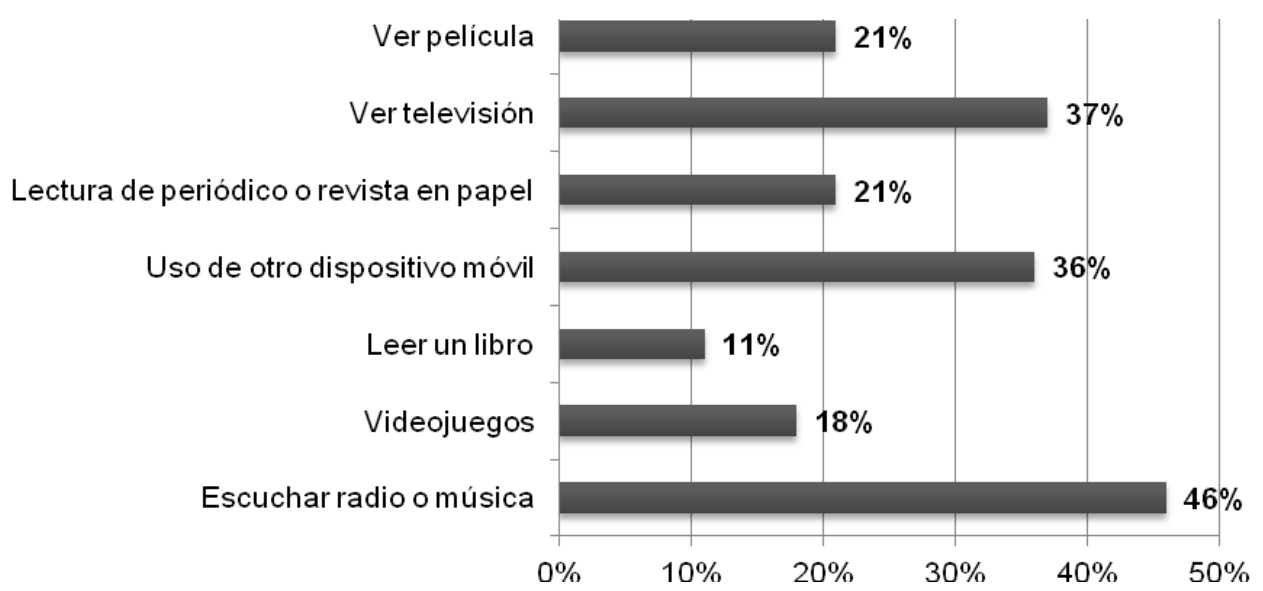

Gráfico 1: Actividades realizadas mientras se usa el móvil. Fuente: Elaboración propia a partir de los datos de Pallete et al., 2012.

El cambio del modelo comunicativo, donde los procesos ya no se basan en una recepción sincrónica de un flujo continuo de contenidos (Aguilera, 2009), ha alterado tanto los contenidos como la industria encargada de su elaboración. La forma en que la televisión se consume ha cambiado, pasando de lo colectivo a lo individual (Arrojo Baliña, 2008), de manera que cada vez se ve más televisión en los dispositivos móviles, lo que provoca que se promueva menos la socialización con el entorno físico inmediato y más la virtual colectiva (muchos portales web de las cadenas movilizan el intercambio de opiniones de los seguidores de sus programas en Facebook y Twitter). A ello se unen otros aspectos: los hogares multipantalla con dispositivos informáticos, smartphones y tabletas son ahora un lugar común (Majó, 2009) y en los espectadores se ha producido un cambio: se han convertido en sus propios programadores gracias a la posibilidad de visionar contenidos a la 
carta (Peris, 2011). En definitiva, pasamos de la hegemonía de la comunicación de masas a través de la televisión a una televisión hecha a la medida de cada individuo, con la posibilidad de llevarla en el bolsillo. Es ni más ni menos que una ventana al mundo móvil e interactiva (Arrojo Baliña, 2008).

El cambio en el universo mediático es conocido por muchos como la "autocomunicación de masas" (Courtois et al., 2012), donde se definen nuevas características para la comunicación: simultánea, masiva, multimodal, autogenerada, autodirigida y autoseleccionada. La interactividad escapa a las funcionalidades habituales de la comunicación de masas de manera que, junto al resto de rasgos señalados, huye de los esquemas recurrentes para definir este tipo de comunicación (Arrojo Baliña, 2008). De hecho, la comunicación interactiva apunta aspectos que serán el signo distintivo de las aplicaciones de segunda pantalla, tales como complementar la programación de televisión y el acceso a informaciones asociadas al contenido ofrecido en la pantalla del televisor (León \& García, 2002).

Como resultado de la referida convergencia multimedia y de la constante búsqueda de atractivos y novedosos productos para los espectadores, las cadenas de televisión hacen esfuerzos por adaptarse a las audiencias jóvenes, las que de forma natural tienen mayor relación con las nuevas tecnologías y las que progresivamente ven menos la televisión convencional, la "primera pantalla", según indican las estadísticas y estudios de audiencia de los últimos tiempos (Arjona Martín, 2009). La respuesta se ha encontrado en la implementación de las innovaciones de última generación, como son las aplicaciones para dispositivos móviles. Sin embargo la industria televisiva, en esta expansión tecnológica y de nuevas pantallas, no se quiere limitar únicamente al espectador juvenil y pretende alcanzar un mercado lo más heterogéneo posible en cuanto a perfil generacional (Fernández de Arroyabe, López \& Peñafiel, 2007), especialmente impulsado por la importante implantación de smartphones y tabletas entre la población adulta.

El visionado compartido de la televisión con otras pantallas no es ninguna novedad: muchos espectadores, sin manejar una aplicación de second screen, navegan por internet al mismo tiempo que están delante del televisor desde hace años, especialmente concentrados en páginas de redes sociales. Algunos estudios cifran esta tarea en casi un 60\% (Holmes et al., 2012), otros en un $86 \%$ (Red Bee Media, 2012a y 2012b). Ello hace necesario una aclaración conceptual de lo que es la actividad de segunda pantalla en relación con la televisión, la cual engloba tres

DOI: ri14.v11i2.568 | ISSN: 1697-8293 | Año 2013 Volumen 11 N² 2 | ICONO14 
acciones distintas (Red Bee Media, 2012a):

- Experiencias de acompañamiento: actividad en un dispositivo de segunda pantalla, diseñada por el creador del servicio o de los contenidos que se están visionando en la televisión como parte del entretenimiento que dispensa. Engloba cualquier experiencia que funciona como contrapartida del consumo de televisión, dirigida en este caso a una segunda pantalla.

- Actividad en segunda pantalla sincronizada con el espacio televisivo que se está viendo en ese instante.

- Visionado dual: podría considerarse la actividad más amplia al intentar definir el uso de la segunda pantalla debido a que engloba cualquier tarea que implique el uso de un dispositivo mientras se ve televisión, ya sea para enviar correos electrónicos, consultar la información meteorológica, etc.

De manera que lo que llamamos aplicaciones de segunda pantalla (también conocidas como second screen, secondary screen y dual screen apps) suponen al menos el desarrollo de dos de estas tres actividades: acompañamiento y visionado dual (dejando como opción la posibilidad de la sincronización, por otra parte más común que la asincronía (Cesar et al., 2009). Siguiendo otra clasificación realizada por Red Bee Media en el primero de sus informes sobre la segunda pantalla (2012a), podemos realizar una distribución razonada de las aplicaciones en su actividad básica: la de acompañamiento. Para ello se van a tener en cuenta dos factores: si están diseñadas para ser usadas frente al televisor o en otro emplazamiento $\mathrm{y}$, en segundo lugar, cómo se va a usar: para el descubrimiento (búsqueda, recomendaciones) o si se vincula al contenido de un programa íntegro, parte de él, aporta extras... La taxonomía resultante del cruce de las circunstancias descritas es ésta:

\section{Tipología de aplicaciones en relación a la función}

\section{de acompañamiento}

\begin{tabular}{|l|l|l|}
\hline & Contenido & Descubrimiento \\
\hline Ante el televisor & Aplicaciones de contenido & Aplicaciones de control \\
& interactivo & remoto \\
& (Control Interaction Apps) & (Remote Control Apps) \\
\hline
\end{tabular}

ICONO14 | Año 2013 Volumen 11 N² 2 | ISSN: 1697-8293 | DOI: ri14.v1 1i2.568 


\begin{tabular}{|l|l|l|}
\hline & Contenido & Descubrimiento \\
\hline Otro emplazamiento & $\begin{array}{l}\text { Aplicaciones de consumo } \\
\text { remoto } \\
\text { (Remote Consumption Apps) }\end{array}$ & $\begin{array}{l}\text { Aplicaciones de dirección } \\
\text { remota } \\
\text { (Remote Management Apps) }\end{array}$ \\
\hline
\end{tabular}

Tabla $n^{\circ}$ 1: Tipología de aplicaciones en relación a la función de acompañamiento.

Fuente: elaboración propia a partir de Red Bee Media, $2012 a$.

Las aplicaciones de contenido interactivo son las que ofrecen obviamente interacción, pero también todo tipo de información adicional y experiencias sociales virtuales junto a otras funcionalidades relacionadas con un espacio televisivo, con el cual habitualmente hay sincronía (existen cuatro tecnologías de interacción sincrónica: por código de tiempo, huellas, marcas de agua y por inicio de sesión vía registro). Los géneros más adecuados para la sincronía de sistemas de televisión social interactiva son los que precisamente incentivan más la conversación con los acompañantes durante su emisión: informativos, series, concursos y retransmisiones deportivas; mientras, los indicados para arquitecturas asíncronas son las películas, documentales y programas musicales (Geerts et al., 2008).

Las aplicaciones de control remoto están diseñadas para emplearse en lugar del tradicional mando a distancia o la guía electrónica de programas (EPG), al igual que éstos tienen como fin encontrar información de un espacio televisivo, configurar un menú, grabar, etc ${ }^{1}$. Las aplicaciones de control remoto dan acceso a los programas de televisión a través de internet, ya sea para actualizarse sobre un contenido o disfrutar de un streaming. Por último, las aplicaciones de dirección remota hacen posible que el espectador pueda enviar órdenes (como grabar) al sistema de televisión de su hogar.

Otras propuestas de clasificación manejan los usos de la segunda pantalla en el entorno televisivo (Cesar et al., 2009):

- Control de contenido (content control) en referencia a las decisiones sobre qué y cómo consumir del contenido televisivo. Algunas subclases de esta categoría son la selección y navegación por el contenido, visionado de material extra, control de vídeos, etc.

- Enriquecimiento de contenido (content enrich): supone una manipulación activa del contenido multimedia. Entre sus subtipos se encuentra la creación, fragmentación y enriquecimiento de contenidos e inclusión de comentarios.

DOI: ri14.v11i2.568 | ISSN: 1697-8293 | Año 2013 Volumen 11 N² 2 | ICONO14 
- Compartir contenido (content sharing) en medios sociales, ya sea tanto para asuntos y aspectos personales como para fragmentos multimedia.

En el marco del modelo comunicativo digital, las aplicaciones de segunda pantalla suponen un paso más allá de la televisión 2.0, materializado éste por las páginas web de las distintas cadenas de televisión que además incluyen la posibilidad del visionado en streaming y pseudostreaming de los contenidos ya emitidos por la primera pantalla, como es el servicio "TVE a la carta" de la web de RTVE y el "Modo salón" del portal de Antena 3 y La Sexta (servicio que, como hemos visto, integran algunos sistemas de aplicaciones de segunda pantalla). Planteadas o no para el uso sincrónico con la programación de la primera pantalla, se trata de una forma de expandir la experiencia del espectador y de mantenerle ante el televisor cuando los ciudadanos están utilizando cada vez menos tiempo para ver programas de televisión, por lo que las emisiones se encuentran también bajo presión y estudian, constantemente, la forma de ofrecer nuevos servicios interactivos y retener así ante sus pantallas, a audiencias que, en otro caso, podrían perderse de forma definitiva (Urretavizcaya Hidalgo, 2008).

Sin embargo, los diseñadores de estas aplicaciones afrontan un gran reto: si la atención no es convenientemente dirigida entre las dos pantallas, el dispositivo móvil podría disminuir más que aumentar la vinculación del espectador con el programa (Holmes et al., 2012).

La primera generación de aplicaciones para tableta sincronizadas con el contenido de una emisión televisiva data de la primavera de 2011; las pioneras fueron dos apps para iPad específicas para las series norteamericanas Anatomía de Grey ${ }^{2}$ (ABC, 2005-) y Bones (Fox, 2005-), donde se ofrecía información adicional relacionada con la serie (perfiles de personajes, curiosidades relacionadas con la trama, etc.), acceso a redes sociales para "apoyar el visionado compartido y teniendo experiencias interactivas sincronizadas con el espacio televisivo al programar contenidos como encuestas y preguntas" (Holmes et al., 2012). Les han seguido otras, como la diseñada para los espectadores británicos de la segunda temporada de la serie norteamericana The Walking Dead ${ }^{3}$ (AMC, 2010-), sincrónica y destinada a mantener un contador de zombis muertos según su asesino y arma empleada, donde además se pueden hacer predicciones y apuestas sobre el número de caminantes que morirán en el capítulo.

La innovación del uso sincronizado de un servicio de segunda pantalla (sea en

ICONO14 | Año 2013 Volumen 11 N² 2 | ISSN: 1697-8293 | DOI: ri14.v1 1i2.568 
tableta, iPad, smartphone, ordenador personal o cualquier otro dispositivo) es la fidelización del espectador con la primera pantalla, la del televisor, donde está teniendo lugar la emisión de la serie o programa al que se vincula la aplicación. Así lo concluye un estudio realizado sobre el nivel de atención visual, cuyos resultados son concluyentes (Holmes et al., 2012): el 60\% del tiempo se destina al televisor, el $30 \%$ a la tableta y un $7 \%$ no se dedica a ninguna de las dos pantallas anteriores, de manera que se puede determinar que la segunda pantalla consigue una considerable cota de interés visual. Además, la inclusión de interactividad con redes sociales en la propia aplicación se ha convertido en tendencia, la cual eclosionará en el futuro como una vía de retroalimentación en el entorno de las relaciones sociales multimedia de usuarios con intereses comunes.

Entre todos los dispositivos, varios estudios avalan a la tableta como el más idóneo para las aplicaciones de segunda pantalla por su fácil, intuitivo y cómodo manejo y carácter poco intrusivo frente al PC o el portátil, a lo que se suman sus reducidas dimensiones y la buena resolución gráfica que las caracteriza. En la mayoría de las ocasiones tienen conexión a internet, lo que permite el enlace a redes sociales, un lugar común en el uso de la red actualmente y que hace posible la interacción social virtual del espectador (Courtois et al., 2012).

Sin embargo, por otro lado, las cadenas de televisión, plataformas y proveedores todavía no han logrado obtener el máximo partido en su relación con los espectadores a través de aplicaciones de segunda pantalla, así como tampoco han conseguido capitalizar el creciente interés y empleo de las mismas (Red Bee Media, 2012c).

Recientemente, la televisión en España ha empezado a hacer uso de las posibilidades que ofrece el empleo de la segunda pantalla con el desarrollo de aplicaciones para diferentes dispositivos y sistemas operativos. Además de nuestro objeto de estudio, Ant 3.0, vamos a destacar "Más Isabel", de TVE, y "Check in Fox", de Fox España.

"Más Isabel"4 es una aplicación de segunda pantalla, diseñada y creada específicamente para la serie Isabel, producida por Diagonal TV (primera temporada emitida en otoño de 2012). Descrita como "una experiencia interactiva en directo" (tal y como se la define en el menú de la app para iPad y tabletas de RTVE), se trata del estreno de TVE en las aplicaciones sincrónicas de segunda pantalla. Con ella experimenta, por primera vez bajo este formato, las posibilidades de la experiencia

DOI: ri14.v1 1i2.568 | ISSN: 1697-8293 | Año 2013 Volumen 11 N² 2 | ICONO14 
interactiva del espectador a través del ordenador (accediendo a través del enlace en la página web de la serie, alojada en el portal de RTVE, que aparece únicamente durante la emisión del capítulo, o directamente en http://www.rtve.es/masisabel) o con la aplicación específica para dispositivos con sistemas operativos Android e iOS.

Permite al usuario ampliar sus conocimientos de hechos y personajes históricos siguiendo el ritmo de cada uno de los capítulos de Isabel, se potencia así el uso simultáneo a partir de una serie de avisos o llamadas que aparecen sobreimpresos en la pantalla del televisor, durante la emisión del capítulo, los cuales alertan de la subida de nuevo contenido. La sincronización del empleo de la aplicación con el seguimiento en directo del capítulo permite poner en juego otra baza: la posibilidad de realizar comentarios en conjunción con otros espectadores bien como usuario anónimo, bien a través del perfil en Facebook o mediante el hashtag de Twitter genérico de la serie o el específico que se defina para cada capítulo.

Una vez en la aplicación, por cualquiera de las vías de acceso señaladas, se observa la pantalla dividida en tres sectores (tal como se observa en la figura 2):

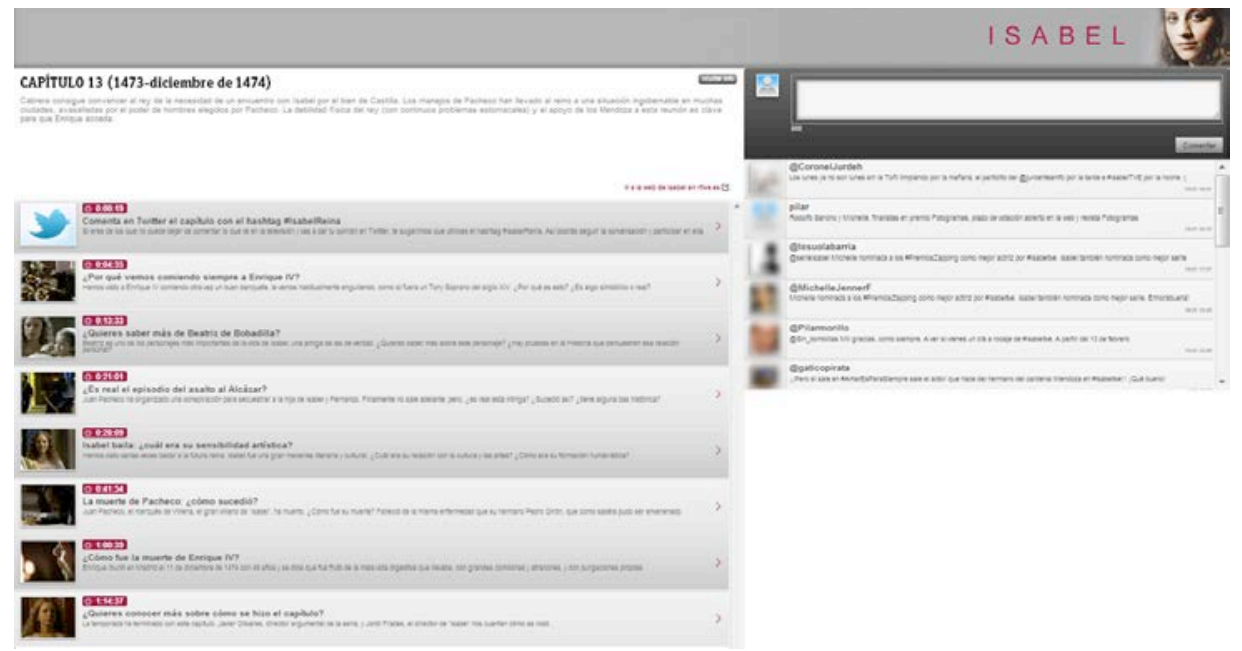

Figura 2: Pantalla principal de "Más Isabel" para el capitulo 13 al finalizar la emisión. Fuente: http://www.rtve.es/masisabel

A la izquierda, en la caja de mayor tamaño, encabeza el número de capítulo, el ámbito temporal en el que se sitúa la acción y una sinopsis argumental. Le siguen abajo los avisos de lo que se va subiendo y cargando conforme la emisión avanza 
(con indicación temporal de acuerdo con la cronometración del capítulo), siempre en estrecha relación con el desarrollo del argumento, y que permiten acceder a informaciones textuales y videográficas relativas a personajes, localizaciones que aparecen, usos y costumbres que se remarquen en el episodio, making of del capítulo, etc., marcadas todas ellas por el aspecto estrictamente histórico-didáctico. Finalmente, a la derecha de la pantalla, se presentan los comentarios que van entrando vía Twitter, a través de la página de la serie en Facebook y también los directamente introducidos en la aplicación de pantalla dual (en este último caso se mantiene el anonimato del autor del comentario). La presencia de la caja de comentarios abre la posibilidad a la interacción social del espectador, como hemos visto una de las características más destacadas del uso de la segunda pantalla.

Otra aplicación en España, además de "Más Isabel" de TVE, es la empleada por Fox España en otoño de 2012: "Check in Fox" (véase figura 3).
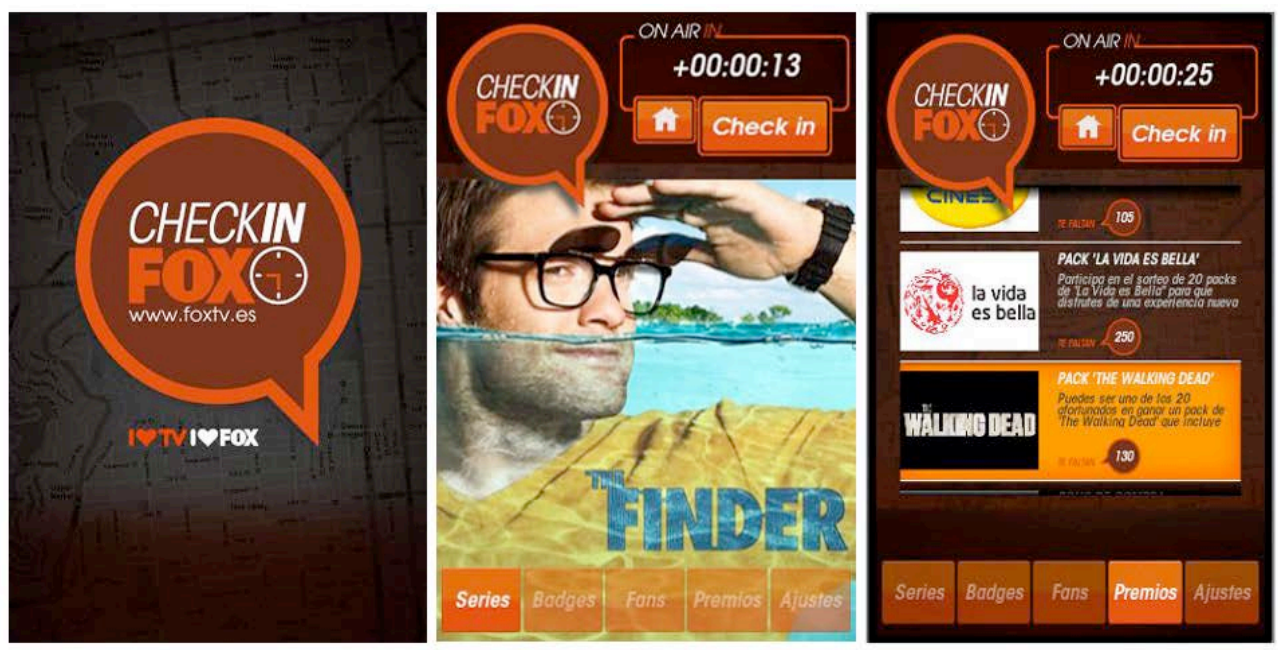

Figura 3: Capturas de pantalla de la aplicación "Check in Fox". Fuente: App Check in Fox

Su naturaleza es distinta a la de TVE: en este caso no se trata de ampliar conocimientos sobre diferentes aspectos relacionados con las series en emisión o la interacción social virtual con otros seguidores, sino que es la primera app en España que permite compartir al espectador su "serieposicionamiento" a través de las redes sociales, lo cual tiene como contraprestación la posibilidad de acceder a premios. Al ver una serie en Fox, se puede hacer check in tantas veces como se desee durante el prime time ${ }^{6}$; la acumulación de veces que se repite esta acción permite conseguir obsequios (cheques regalo, entradas de cine, packs promocionales, etc.)

DOI: ri14.v11i2.568 | ISSN: 1697-8293 | Año 2013 Volumen 11 N² 2 | ICONO14 
y descuentos en establecimientos asociados a la promoción. Se trata de un sistema de puntos, los cuales se acumulan a lo largo de la semana para, al final de la misma y en función de los conseguidos, optar al sorteo de los premios ${ }^{7}$. De esta manera, "Check in Fox" plantea un uso distinto de la segunda pantalla, en este caso como sistema de recompensa al espectador por el seguimiento de las series emitidas por Fox España.

La designación de la aplicación de segunda pantalla del grupo Antena 3 con el apellido "3.0" apunta su asociación con "lo nuevo y a innovación tecnológica, en definitiva, a futuro. Por ello, algunos medios han adoptado la denominación 3.0 para presentar una nueva etapa de desarrollo que no tiene nada que ver con la filosofía 3.0 aplicada a la Red" (Jódar Marín, Polo Serrano \& Jiménez Marín, 2010). Pero no es sólo eso, se vincula directamente con el itinerario de migración hacia la convergencia digital emprendida por Antena 3 hace algunos años con el proyecto Antena 3.0, a través del cual la cadena ha sido pionera en tener canal propio en Youtube, ofrecer acceso gratuito a capítulos íntegros y preestrenar sus series en internet, pionera en España en emitir en directo a través de redes de telefonía 3G, entre otros hitos.

La aplicación gratuita Ant 3.0 está diseñada para dispositivos móviles con los sistemas operativos Android e iOS y ha sido desarrollada por la agencia española de marketing La Moderna ${ }^{8}$ y la compañía británica Intrasonics ${ }^{9}$, con el patrocinio tecnológico de Movistar. La Moderna destaca por su especialización en el desarrollo de nuevos conceptos de comunicación en relación con las nuevas tecnologías, y tiene los derechos de Interaction para la Península Ibérica y Latinoamérica ${ }^{10}$.

Interaction, como se conoce al sistema de comunicación que emplea Ant 3.0, se basa en el reconocimiento de la señal de audio de los programas de Antena 3. Se trata de una tecnología watermarking o de marca de agua, lo que implica que se introduce en un sonido determinado del programa en emisión (ya sea música, diálogo, etc.) una serie de etiquetas acústicas en formato ultrasonido, por lo tanto fuera del margen de frecuencias audibles por el ser humano. Éstas son detectables por el micrófono del dispositivo móvil donde se ha instalado la aplicación Ant 3.0. e incluyen una instrucción codificada que se transforma en contenido audiovisual cuando la tableta, el smartphone, iPad o iPhone captan el sonido. Ello permite que el espectador se convierta en agente activo con los espacios de Antena 3, recibiendo información adicional de forma sincrónica a la emisión y con la que puede rea-

ICONO14 | Año 2013 Volumen 11 N² 2 | ISSN: 1697-8293 | DOI: ri14.v1 1i2.568 
lizar diferentes acciones: responder a preguntas a modo de cuestionario, acceder a contenidos especiales en formato textual o videográfico, entre otras.

Su funcionamiento es éste: tras la descarga en el dispositivo móvil, cada vez que se emita un programa interactivo, se avisa al usuario mediante una notificación emergente de que puede abrir la aplicación para recibir contenidos. Se debe mantener abierta la aplicación de segunda pantalla durante la emisión del programa 0 serie de su interés. En la pantalla de la tableta o el teléfono, según se vayan dando las etiquetas acústicas, irán apareciendo eventos (llamadas textuales) a través de los cuales se accede a contenidos adicionales al visionado en el televisor.

Las posibilidades interactivas que se ofrecen son variadas: recepción de información y vídeos exclusivos del programa o serie que se está siguiendo en ese momento, información de ayuda, expresar opiniones, acceso a contenido multimedia no emitido, making of de los programas, encuestas interactivas... Todos estos contenidos se ofrecen bajo el formato de eventos, como se ha señalado, los cuales pueden están vinculados con el programa en curso o también asociados a spots publicitarios concretos emitidos durante la pausa publicitaria.

Los contenidos audiovisuales se ofrecen en dos formatos: streaming (se recibe una notificación sobre la posibilidad de visualizar vídeos y/o fotografías; si se acepta, el contenido se reproduce automáticamente en el dispositivo) y descarga directa (el contenido se almacena en una carpeta; la reproducción es automática $\mathrm{y}$, frente a la otra alternativa, se puede visualizar tantas veces como se desee). La aplicación proporciona material audiovisual, enlaces a páginas web (esencialmente los perfiles en redes sociales de los programas de Antena 3: Facebook y Twitter) relacionadas con lo que se emite en ese momento. Para algunas series se han realizado iniciativas, como el acceso a un ejemplar del primer guión de Gran Hotel firmado por la actriz protagonista, Amaia Salamanca, entre los usuarios que se descargasen la aplicación y respondieran correctamente a las preguntas formuladas durante la emisión del primer capítulo de la segunda temporada. Para la descarga del guión, desde la web de la serie, se proporcionó una clave a todos los espectadores que acertaron todas las preguntas. Los contenidos exclusivos son accesibles también a través del modo salón de Antena 3 hasta 48 horas después de la emisión del programa o serie.

La aplicación se lanza el 27 de febrero de 2012 con El hormiguero; se alcanzaron más de medio millón de contenidos servidos el día de su estreno, lo cual habla por

DOI: ri14.v11i2.568 | ISSN: 1697-8293 | Año 2013 Volumen 11 N² | ICONO14 
sí mismo de la buena acogida entre los usuarios, tanto de Android como de iOS (98.999 y 82.740 descargas, respectivamente), pero hay más datos: en su primera semana de funcionamiento ha registrado 2.200 .000 contenidos servidos y más de 200.000 descargas de la aplicación. Asimismo, Ant 3.0 ha ocupado durante esta semana las dos primeras posiciones del ranking de las aplicaciones más descargadas de los dos sistemas operativos más importantes del mercado tecnológico.

Desde el lanzamiento de Ant 3.0, el promedio de contenidos servidos (vídeos, audios, imágenes, preguntas, enlaces...) se ha acercado a los 450.000. El día del estreno registró el máximo número de contenidos con 511.742, en el que también se produjo el pico (medidos en franjas de 5 minutos) más elevado con cerca de 63.000 descargas (62.963). Ese momento se dio a las 21:45 horas (Grupo Antena 3, 2012).

El estreno en marzo de 2012 de la tercera temporada de Los protegidos se sumó a la aplicación; también lo hizo posteriormente la primera de Luna, el misterio de Calenda, en abril. De esa manera, de forma progresiva se han ido sumando distintos programas, series y espacios como Tu cara me suena, Gran Hotel, El barco, Fenómenos, Elecciones catalanas 2012, etc., junto a otras acciones promocionales de Antena 3, recientemente fusionada con La Sexta, como Bienvenida al 2013.

Tras alcanzar más de 2 millones de contenidos servidos y superar las 200.000 descargas, a principios de octubre de 2012 se presenta una segunda versión de la aplicación.

Ant 3.0 sirve contenidos de segunda pantalla para espacios de periodicidad semanal y eventos especiales, los cuales son consultables en el menú "programas" de la aplicación. Otra de las opciones, "preferencias", permite que el usuario elija de qué programas desea recibir notificaciones para seguir las sesiones de segunda pantalla. La aplicación también cuenta con un asistente que en siete pasos explica en qué consiste y su funcionamiento:

1. Ant 3.0 es una aplicación que te permite interactuar y recibir contenidos totalmente exclusivos en tu dispositivo al mismo tiempo que disfrutas de tu serie o programa favorito en directo en Antena 3.

2. En la primera pantalla de la aplicación encontrarás la lista de las emisiones interactivas. Consulta esta pestaña para saber cuándo se emitirán los próximos programas con contenido interactivo para Ant 3.0.

3. Una vez abierta la aplicación, pulsa la pestaña de Programas para acceder al contenido interactivo de cada programa o serie. Pulsa Ajustes para dejar de se-

ICONO14 | Año 2013 Volumen 11 N² | ISSN: 1697-8293 | DOI: ri14.v1 1i2.568 
guir algún programa en concreto. Pulsa Inicio para regresar a la lista de próximas emisiones y al asistente.

4. Recibirás un aviso e invitación a interactuar con la serie o programa que tienes activado justo antes de su emisión.

5. Una vez aceptada la invitación, ya está todo listo para que recibas audios, vídeos, imágenes, enlaces y todo tipo de contenido en exclusiva. Y aún más. Con Ant 3.0 podrás participar e interactuar en directo con la emisión en televisión.

6. En la pestaña Programas puedes consultar los contenidos recibidos durante la última emisión pulsando el botón Ver historial que encontrarás bajo la descripción de cada programa o serie.

7. A través del icono de la esquina superior derecha, dentro de cada programa, podrás compartir los contenidos interactivos en redes sociales y podrás dejar de seguir una emisión en curso. También puedes configurar los programas en los que quieres participar en la pestaña de ajustes.

Para profundizar en el conocimiento de la aplicación Ant 3.0, se ha seguido una sesión completa con uno de los programas de Antena 3 a los que se vincula: la serie Gran Hotel, concretamente la que acompañó a la emisión del 22 de enero de 2013 con la que debutó la tercera temporada. Baile de máscaras, como se titula el capítulo, fue la segunda emisión más vista del prime time de ese martes (con 2.626.000 espectadores de media y $14,2 \%$ de share) tras el film Colombiana, estreno de La Sexta.

Al abrir la aplicación, aparece el menú de inicio donde están listadas las próximas emisiones con cobertura de segunda pantalla con Ant 3.0.; tras escoger Gran Hotel, con indicador naranja que apunta el funcionamiento del servicio, se eligió la opción "participar", la cual accionó una pantalla emergente en la que se nos solicitaba confirmación, tal como se muestra en la siguiente secuencia de capturas (figura 4): 


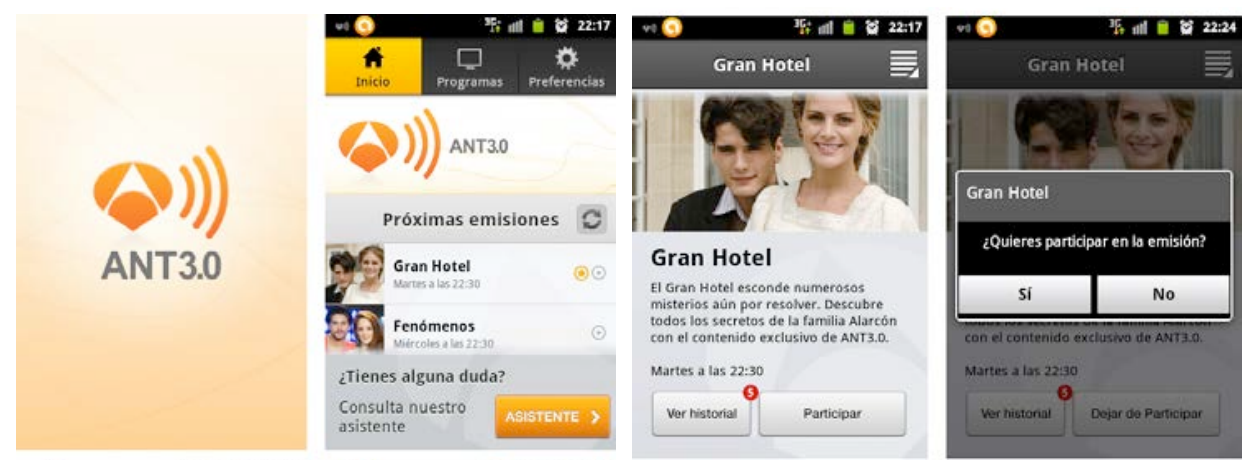

Figura 4: Inicio de la sesión de segunda pantalla para Gran Hotel. Fuente: Aplicación Ant 3.0

A lo largo de la emisión se generaron diecisiete contenidos de cinco tipos: vídeos (cinco), texto (tres), imagen (dos), web (dos) y preguntas (cinco), todos ellos en estrecha relación con el desarrollo del argumento del episodio, ofreciendo incluso avances tanto de lo que estaba por ocurrir en el capítulo en curso como en relación al próximo.

La totalidad de los vídeos ofrecidos fue vía streaming; se solicitaba confirmación para su visionado y no entorpecer el seguimiento del capítulo en la primera pantalla, si así lo desea al espectador, tal como se muestra en la figura 5.

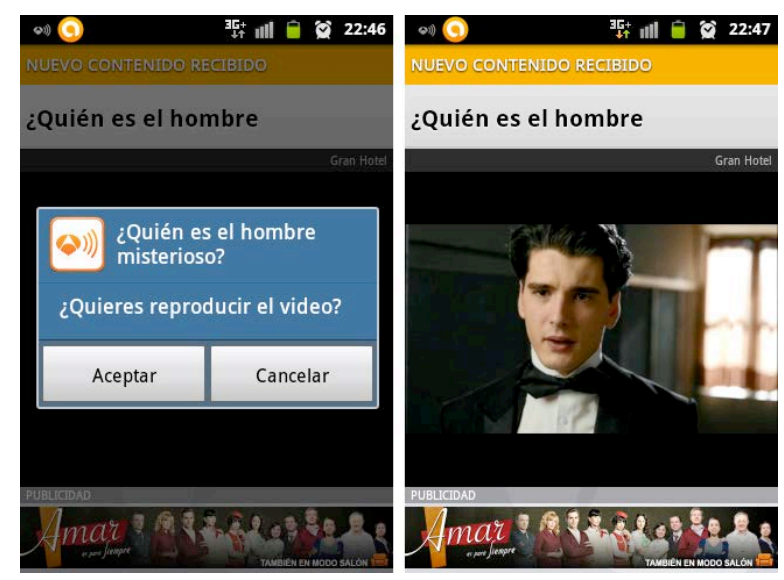

Figura 5: Vídeo ¿Quién es el hombre misterioso?. Fuente: Aplicación Ant 3.0

Todos los vídeos ofrecidos tienen naturaleza de avance: los dos primeros se adelantaron unos minutos al comienzo de la emisión del capítulo, el cual tuvo lugar a las 22:52 h.: ¿Quién es el hombre misterioso? (22:46) y En este capítulo (22:50). El tercero se ofreció durante la publicidad, Avance: Beatriz y Doña Teresa (23:30), un anticipo de lo que en breve se iba a ver; el cuarto se recibió con el episodio en 
emisión: Avance: Una nueva pista (23:48) y el último cuando ya había concluido: Las incógnitas de Alicia (00:20). En todos los casos la extensión de los vídeos no sobrepasaba los ochenta segundos.

Los contenidos textuales ofrecidos tienen igualmente naturaleza de avance, terminando con una pregunta que interpela a la curiosidad del espectador. La tipografía empleada es de gran tamaño, lo que permite una adecuada lectura y hace necesario, por otra parte, el empleo de la barra lateral de deslizamiento para su completa lectura. En todos los casos, los textos van encabezados por una imagen alusiva a su contenido. Veamos todo ello reflejado en la figura 6:

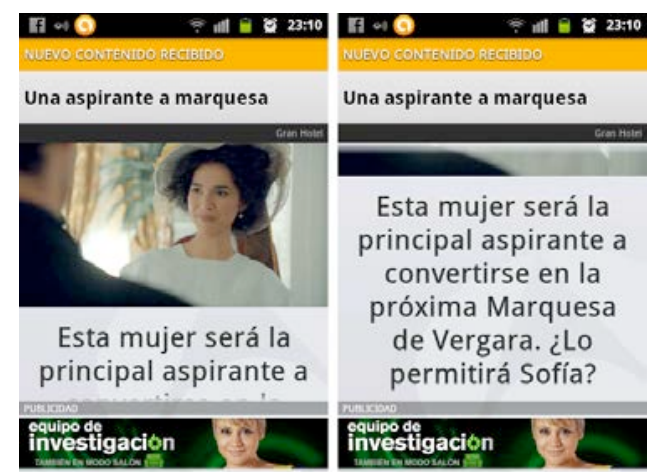

Figura 6: Contenido textual: Una aspirante a marquesa. Fuente: Aplicación Ant 3.0

De los tres contenidos de texto generados, uno de ellos avisaba al espectador: "permanece atento a la publicidad y recibirás en primicia escenas exclusivas de los próximos capítulos de Gran Hotel sólo para usuarios de ANT3.0" (Durante la publicidad..., 23:26). Durante los minutos de interrupción publicitaria se ofreció el vídeo de avance Beatriz y Doña Teresa ya comentado.

Las fotografías que constituyen por sí mismas contenido tienen, como la mayor parte de lo ya señalado, carácter de avance. De las dos que se ofrecieron, una tenía relación directa con el capítulo en curso: la primera sirvió de presentación de un nuevo personaje cuya participación en la trama era en ese momento desconocida por el espectador (¿Quién es este hombre?, 23:16) y la segunda avisa del regreso de otro (Belén regresará al Gran Hotel, 23:57). En todos los casos se permite la descarga de la imagen en la carpeta de la aplicación en el dispositivo, como muestra la figura 7: 


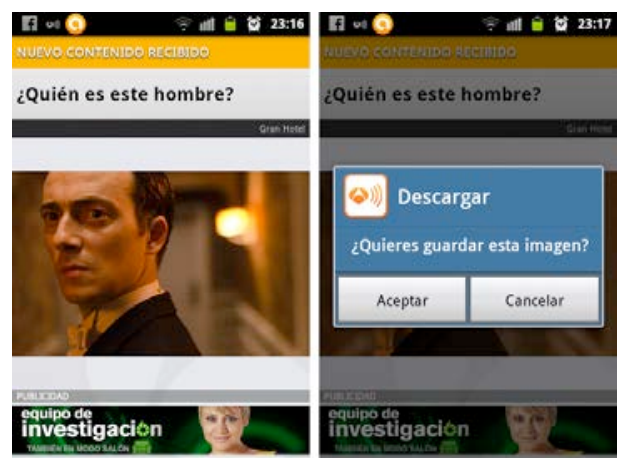

Figura 7: Contenido fotográfico. Fuente: Aplicación Ant 3.0

Otro tipo de informaciones generadas son los accesos web, en este caso a la página de la serie en Facebook y al perfil en Twitter para animar al espectador a seguir la serie en esos contextos e introducir sus comentarios, opiniones, etc.

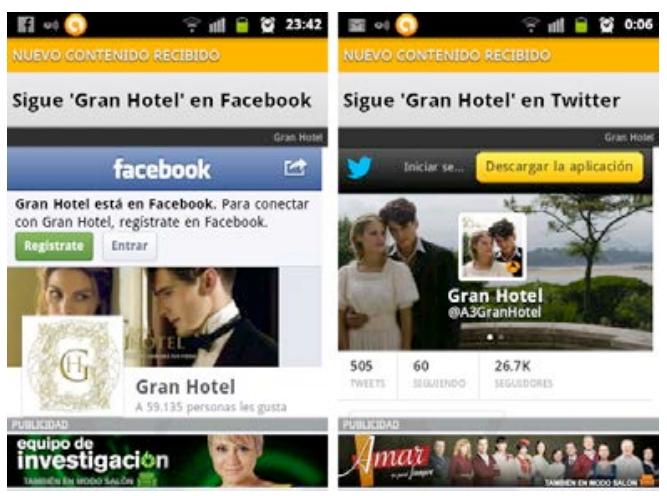

Figura 8: Sigue Gran Hotel en Facebook y Twitter. Fuente: Aplicación Ant 3.0

Por último se encuentran las preguntas directas al espectador con una batería cerrada de respuestas en formato test. Junto a los vídeos, es el contenido que fue más numeroso y que no aparece registrado en el historial de la sesión que ofrece la aplicación. De todo lo ofrecido, es lo que más refuerza la interactividad ya que la contestación del seguidor recibe instantáneamente la solución (correcto 0 incorrecto). Si la respuesta es correcta, ésta aparece argumentada y, en caso incorrecto, el espectador tiene notificación de que si acierta la próxima recibirá pistas adicionales, a modo de recompensa por su sagacidad e intuición. Véase en la figura 9 ambos supuestos. 


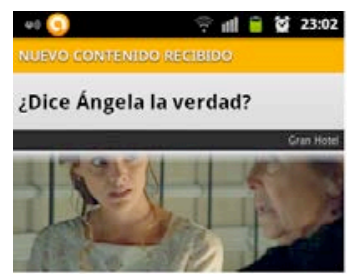

¿Dice Ángela la verdad?

(1) Si

(2) No, conoce a ese hombre

3 No, miente sobre el traje

investigacion
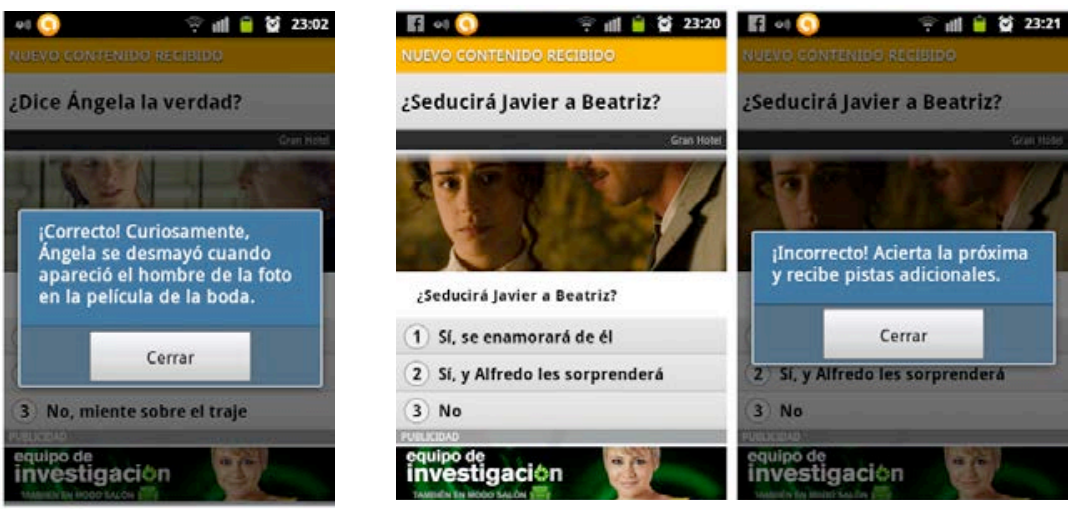

Figura 9: Cuestionario. Fuente: Aplicación Ant 3.0

En los cuatro primeros contenidos de pregunta se interroga al espectador sobre incógnitas de la trama a los que se dará resolución en los minutos posteriores. En el quinto, el último, con el episodio ya concluido, se indaga acerca de la cuestión que ha dejado abierta el final del capítulo: se ha producido un disparo pero se desconoce la identidad de la víctima (Alguien ha muerto, ¿quién?, 00:18). De esta manera, las preguntas emergentes se apuntan al importante componente de intriga de la serie, potenciando la interactividad del espectador, el cual se asimila al detective que desentraña algunos de los secretos de la familia Alarcón (de la misma forma que hace uno de los personajes, el inspector Ayala, y otro de los aparecidos en la segunda temporada: una joven Agatha Christie de paso por el Gran Hotel). Su puntería al responder, además, tiene contraprestación en forma de más pistas y contenidos.

Para conocer el orden en que se fueron dando los eventos, se presenta la cronología de la sesión:

- 22:46 h., ¿Quién es el hombre misterioso? (vídeo).

- 22:50 h., En este capítulo (vídeo).

- 22:53 h., La sorpresa de Don Ernesto (texto).

- 23:02 h., ¿Dice Ángela la verdad?, (cuestionario).

- 23:10 h., Una aspirante a marquesa (texto).

- 23:16 h., ¿Quién es este hombre? (fotografía).

- 23:20 h., ¿Seducirá Javier a Beatriz? (cuestionario).

- 23:26 h., Durante la publicidad... (texto).

- 23:30 h., Avance: Beatriz y Doña Teresa (vídeo).

- 23:37 h., ¿Hará Alicia lo que pide Garrido? (cuestionario). 
- 23:41 h., Sigue Gran Hotel en Facebook (enlace web).

- 23:48 h., Avance: una nueva pista (vídeo).

- 23:57 h., Belén regresará al Gran Hotel (fotografía).

- 00:02 h., ¿Coincidirán Fernando y Andrés? (cuestionario).

- 00:06 h., Sigue Gran Hotel en Twitter (enlace web).

- 00:18 h., Alguien ha muerto, ¿quién? (cuestionario).

- 00:20 h., Las incógnitas de Alicia (vídeo).

En el historial de contenidos de la sesión en la aplicación no aparecen todos los aquí señalados, quedan excluidas las preguntas directas al espectador al que se le demanda una respuesta. El histórico permanece accesible y consultable a lo largo de toda la semana, desapareciendo el día de la emisión; en él, las informaciones están clasificadas por iconos que identifican su tipología (vídeo, web, imagen o texto), así como la fecha y la hora tal en que se dieron, como se muestra en la figura 10:

\begin{tabular}{|c|c|c|c|}
\hline \multicolumn{2}{|r|}{ Historial de Gran Hotel } & \multicolumn{2}{|c|}{ Historial de Gran Hotel } \\
\hline & ulsar para actualizar... & & $\begin{array}{l}\text { Las incógnitas de Alicia } \\
\text { martes } 22 \text { de enero a las } 23: 19\end{array}$ \\
\hline & $\begin{array}{l}\text { ¿Quién es este hombre? } \\
\text { martes } 22 \text { de enero a las } 22: 16\end{array}$ & WWWW. & $\begin{array}{l}\text { Sigue 'Gran Hotel' en Twit... } \odot ~ \\
\text { martes } 22 \text { de enero a las 23:06 }\end{array}$ \\
\hline & $\begin{array}{l}\text { Una aspirante a marquesa } \\
\text { martes } 22 \text { de enero a las } 22: 09\end{array}$ & & $\begin{array}{l}\text { Belén regresará al Gran H... } \odot ~ \\
\text { martes } 22 \text { de enero a las 22:56 }\end{array}$ \\
\hline$T$ & $\begin{array}{l}\text { La sorpresa de don Ernesto } \\
\text { martes } 22 \text { de enero a las } 21: 52\end{array}$ & fing & $\begin{array}{l}\text { Avance: Una nueva pista } \\
\text { martes } 22 \text { de enero a las 22:48 }\end{array}$ \\
\hline fint & $\begin{array}{l}\text { En este capítulo... } \\
\text { martes } 22 \text { de enero a las } 21: 47\end{array}$ & WWWW. & $\begin{array}{l}\text { Sigue 'Gran Hotel' en Face... } \odot \text { } \\
\text { martes } 22 \text { de enero a las } 22: 40\end{array}$ \\
\hline$f^{\prime r}$ & $\begin{array}{l}\text { ¿Quién es el hombre mist... } \\
\text { martes } 22 \text { de enero a las } 21: 45\end{array}$ & fors & $\begin{array}{l}\text { Avance: Beatriz y doña Te... } \\
\text { martes } 22 \text { de enero a las } 22: 29\end{array}$ \\
\hline
\end{tabular}

Figura 10: Capturas del historial de Gran Hotel durante la sesión realizada.

Fuente: Aplicación Ant 3.0

Durante la transmisión del capítulo, elementos gráficos emergentes sobreimpresionados en la esquina superior izquierda pantalla del televisor anunciaban que se trataba de una emisión interactiva, invitando al espectador a descargarse la aplicación y convertirse así en un personaje más. 


\section{Discusión}

En el entorno actual de convergencia multimedia y de pantallas, el modo en el que la televisión se ve ha cambiado: no se consume únicamente a través del televisor, la pantalla primera y tradicional, sino que los dispositivos móviles han puesto en bandeja, a través del desarrollo de las telecomunicaciones e internet, la posibilidad de acceder tanto a la emisiones de televisión como a contenidos y productos adicionales que complementan la experiencia televisiva. En este contexto, las aplicaciones de segunda pantalla para dispositivos móviles se encuentran en pleno auge como fórmula para lograr la ansiada interactividad del medio, responder a la demanda de más capacidad de acción y contenidos por parte de los espectadores.

Actualmente, la implantación de la segunda pantalla en España está en su estado inicial, al igual que también está en ciernes su desarrollo definitivo y explotación de todo su potencial. Los dos ejemplos más relevantes de aplicaciones de contenido son "Más Isabel", de TVE y específica para la serie Isabel, y con mayor recorrido y amplitud se encuentra Ant 3.0, del grupo Antena 3.

Ant 3.0 es una aplicación de segunda pantalla completa que engloba tres acciones al mismo tiempo (a su vez, trío de las distintas variedades de second screen): acompañamiento, visionado dual y sincronizado. Precisamente por este último aspecto, vinculado con el hecho de que está diseñada para emplearse ante el televisor por su tecnología de marcas de agua y cuyo contenido se vincula con lo que se está siguiendo en la pantalla del televisor, se trata de una aplicación de contenido interactivo (control interaction app). La alternativa que Ant 3.0 supone hacer posible de una forma real la comunicación bidireccional entre televisión y espectadores, lo que redunda en un refuerzo de la fidelización de éstos. Como la sesión de Gran Hotel seguida con Ant 3.0 ha demostrado, la amplia tipología de contenidos que se ofrecen (vídeos, texto, fotos, enlaces web a redes sociales $\mathrm{y}$, especialmente, las preguntas) enganchan al espectador a la intriga en que se desarrolla la trama con los secretos de los Alarcón, la familia protagonista, proporcionando al seguidor pistas y avances de lo que va a suceder, así como hacerle partícipe del propio acto de investigación y descubrimiento de los misterios, como si del propio personaje del detective Ayala se tratase. Los cuestionarios emergentes, que no aparecen en el histórico, y por lo tanto son netamente experiencia sincrónica en directo, recompensan al espectador sagaz con nuevas pistas, junto a contenidos inéditos y ex-

DOI: ri14.v11i2.568 | ISSN: 1697-8293 | Año 2013 Volumen 11 N² 2 | ICONO14 
clusivos. Las aplicaciones interactivas en la primera pantalla (formato botón rojo, por ejemplo) tienden a interrumpir, más que a enganchar. No obstante, se muestra cada vez más interés por el potencial de las aplicaciones de segunda pantalla para vincularse con el contenido televisivo: votando o respondiendo a breves preguntas (Courtois et al., 2012), tal como sucede con el contenido de cuestionario cerrado de Ant 3.0.

La tendencia creciente en las aplicaciones de segunda pantalla de contenido interactivo es la inclusión de la interactividad, valga la redundancia, con redes sociales, las cuales suelen incluirse como parte expresa en el diseño de la app (tal como se ha visto en el breve análisis formal de "Más Isabel"); sin embargo, en el caso de Ant 3.0 no forma parte de la arquitectura de la aplicación, sino que como uno más de los eventos se invita al espectador a comentar en la página en Facebook o en el perfil en Twitter de la serie. Sea como fuere, se ejemplifica una de las características de la nueva televisión: el visionado se hace cada vez más individual mientras que se comparte de forma más global. Paradójicamente, como hemos visto a lo largo de esta investigación y se demuestra con esto, el contexto multipantalla induce simultáneamente a lo privado y lo público (la interacción social virtual).

Ant 3.0 se inscribe en la progresiva carrera del grupo Antena 3 en la integración de nuevas tecnologías, canales y servicios para sus espectadores, siendo la cadena pionera en España respecto a sus iniciativas en este sentido.

\section{Notas}

[1] Para conocer más detalles relativos a prototipos de PDA con estas funciones y experiencias piloto véase Tsekleves et al., 2007.

[2] Vídeo explicativo del funcionamiento de la aplicación: http://www.youtube.com/ watch?v=zqbXBcUbmYw.

[3] Vídeo explicativo de su funcionamiento: http://vimeo.com/37516900.

[4] Vídeo explicativo de su funcionamiento: http://www.rtve.es/alacarta/videos/isabel/asi-funciona-second-screen-isabel-mas-isabel/1522509/

[5] http://checkinfox.es/

ICONO14 | Año 2013 Volumen 11 Nº 2 | ISSN: 1697-8293 | DOI: ri14.v1 1i2.568 
[6] Lunes a viernes, de 21:30 a 23:15, sábado de 22:00 a 23:45 y domingo de 22:00 a 00:30 horas.

[7] Vídeo promocional: http://www.youtube.com/watch?\&V=eAZ0BA052UQ

[8] http://www.agenciamoderna.es

[9] http://www.intrasonics.com

[10] http://www.marketingdirecto.com/especiales/apps-especiales/antena-3-y-el-hormigueroestrenan-ant3-0-a-nivel-mundial-con-la-colaboracion-de-movistar/

\section{Referencias}

Adelantado, E. \& Martí, J. (2011). Contenidos audiovisuales y televisivos para dispositivos móviles: una aproximación al mercado español. AdComunica. Revista de Estrategias, Tendencias e Innovación en Comunicación, 1 (1), 99 113.

Aguilera Moyano, M. de (2009). Innovación, prácticas culturales y contenidos audiovisuales. Una introducción. En M. de Aguilera Moyano \& M. Meere (coords.), Una tele en el bolsillo. La televisión en el teléfono móvil: contenidos, formatos, audiencias (pp. 9-16). Málaga: Círculo de Estudios Visuales Ad Hoc. Arjona Martín, J. B. (2009). Los nuevos canales audiovisuales basados en web: rtve.es. Icono 14. Revista de Comunicación y Nuevas Tecnologías, 8 (1), 98113. doi: $10.7195 /$ ri14.v8i1.283

Arrojo Baliña, M. J. (2008). La configuración de la televisión interactiva: de las plataformas digitales a la TDT. La Coruña: Netbiblio.

Cebrián Herreros, M. (2004). Modelos de televisión: generalista, temática y convergente con Internet. Barcelona: Paidós.

Cesar, P., Bulterman, D. C. A. \& Jansen, J. (2009). Leveraging user impact: an architecture for secondary screens usage in interactive television. Multimedia Systems, 15 (3), 172-142. doi: 10.1007/s00530-009-0159-z

Courtois, C. \& D`Heer, E. (2012). Second screen applications and tablets users: constellation, awareness, experience, and interest (pp. 153156). EuroITV'12. 10th European Interactive TV Conference. Berlín. doi: $10.1145 / 2325616.2325646$

DOI: ri14.v11i2.568 | ISSN: 1697-8293 | Año 2013 Volumen 11 N² 2 | ICONO14 
Fernández de Arroyabe, A., López, N. \& Peñafiel, C. (2007). Las aportaciones de la tecnología digital al nuevo modelo de programación y de consumo de televisión. En C. Peñafiel Saiz (coord.), Transformaciones de la radio y la televisión en Europa (pp. 177-187). Bilbao: Universidad del País Vasco.

Geerts, D., Cesar, P. \& Bulterman, D. (2008). The implications of Programs Genres for the Design of Social Television Systems. En UXTV'08. Proceedings of the 1st international conference on Designing interactive user experiences for TV and video (pp.71-80). Sillicon Valley (California). doi: $10.1145 / 1453805.1453822$

Grupo Antena 3 (2012): Informe de Ant. 3.0. (informe de 7 de marzo). Recuperado de http://www.grupoantena3.com/a3document/2012/05/24/ DOCUMENTS/00013/00013.pdf

Holmes, M. E., Josephson, S. \& Carney, R. E. (2012). Visual attention to television programs with a second-screen application. En ETRA'12. Proceedings of the Symposium on Eye Tracking Research and Applicatons (pp. 397-400). Santa Bárbara, California. doi: 10.1145/2168556.2168646

Jódar Marín, J. A., Polo Serrano, D. \& Jiménez Marín, G. (2010). ¿Dónde quedó el 2.0? El falso concepto de la televisión 3.0. Icono 14. Revista de Comunicación y Nuevas Tecnologías, 8 (3), 251-264. doi: 10.7195/ri14.v8i3.239

León, B. \& García Avilés, J. A. (2002). Los retos de la implantación de la televisión interactiva a la luz de su propia historia. Zer. Revista de Estudios de Comunicación, 7 (13), 95-113. Recuperado de http://www.ehu.es/zer/ hemeroteca/pdfs/zer13-06-leon.pdf

Majó, J. (2009). La televisión pública: financiación y contenidos. En M. Francés i Domenec (coord.), Hacia un nuevo modelo televisivo. Contenidos para la televisión digital (pp. 227-240). Barcelona: Gedisa.

Pallete, A., Jiménez, R., Paley, W. \& Mack, A. (2012). 15 ways mobile will change our lives. JWT Intelligence. Recuperado de http://www.jwtintelligence.com/ wp-content/uploads/2012/03/F_PUBLIC_15_Ways_Mobile_Will_Change_0ur_ Lives_03_22_12.pdf

Peris, A. (2011). Los discursos sobre la calidad en los contenidos audiovisuales para internet y móvil. En M. Francés i Domenec (coord.), Contenidos y formatos de calidad en la nueva televisión (pp. 129-160). Madrid: Instituto RTVE.

ICONO14 | Año 2013 Volumen 11 N² 2 | ISSN: 1697-8293 | DOI: ri14.v1 1i2.568 
Red Bee Media (2012a): Second screen series, paper 1: setting the scene. Recuperado de http://www.redbeemedia.com/sites/all/files/downloads/ second_screen_series_paper_1_whitepaper_red_bee_media.pdf.

Red Bee Media (2012b): Second screen series, paper 2: dual screen consumers. Recuperado de http://www.redbeemedia.com/sites/all/files/downloads/ second_screen_series_paper_2_whitepaper_red_bee_media.pdf.

Red Bee Media (2012c): Second screen series, paper 3: the commercial response. Recuperado de http://www.redbeemedia.com/sites/all/files/downloads/ second_screen_series_paper_3_whitepaper_red_bee_media.pdf.

Tsekleves, E., Cruickshank, L., Hill, A., Kondo, K. \& Whitman, R. (2007). Interacting with Digital Media at Home via Second Screen. En 9th IEEE International Symposium on Multimedia Workshops 2007 (pp. 201-206 Taichung (Taiwan). doi: 10.1109/ISM.Workshops.2007.42 Urretavizcaya Hidalgo, M.(2008). La nueva televisión digital en el universo multimedia. San Sebastián: Universidad de Deusto. 